\title{
Onychomycosis due to Aspergillus niger without black nail discoloration: A case report
}

\author{
Funda Tamer ${ }^{1}$, Mehmet Eren Yuksel ${ }^{2}$
}

${ }^{1}$ Department of Dermatology, Medical Park Hospital, Ankara, Turkey, ${ }^{2}$ Department of General Surgery, Devrek State Hospital, Zonguldak, Turkey

Corresponding author: Dr. Funda Tamer, E-mail: fundatmr@yahoo.com

Sir,

A 39-year-old Caucasian male presented with a 5-year history of thickened toenail. The patient admitted that he used topical isoconazole nitrate twice daily for two months, without any improvement. The past medical history was unremarkable. The physical examination revealed subungual hyperkeratosis and onycholysis on the lateral side of the first toenail, onychoschizia of the second and fourth toenails, subungual hyperkeratosis of the fifth toenail. Our initial diagnosis was distal lateral subungual onychomycosis (Fig. 1). Therefore, we performed mycological culture of nail clippings of the first toenail using Sabouraud's dextrose agar. Aspergillus niger was isolated (Fig. 2).

Black pigmentation may be observed in the nails due to conidia of Aspergillus niger [1]. It is a diagnostic clue for onychomycosis due to Aspergillus niger, however, we didn't observe any pigmentation in our patients toenail.

The serum levels of aspartate aminotransferase, alanine aminotransferase and creatinine were all within normal limits. The patient was treated with oral terbinafine $250 \mathrm{mg}$ daily and topical amarolfine $5 \%$ lacquer once weekly for the last two months. Satisfactory clinical improvement was achieved. Antifungal therapy was continued and regular follow up was recommended.

Onychomycosis is the fungal infection of the nail. The causative agent of onychomycosis is usually a dermatophyte which is called tinea unguium. However, nondermatophyte molds like Aspergillus, Acremonium, Scopulariopsis and Fusarium are responsible for $2 \%$

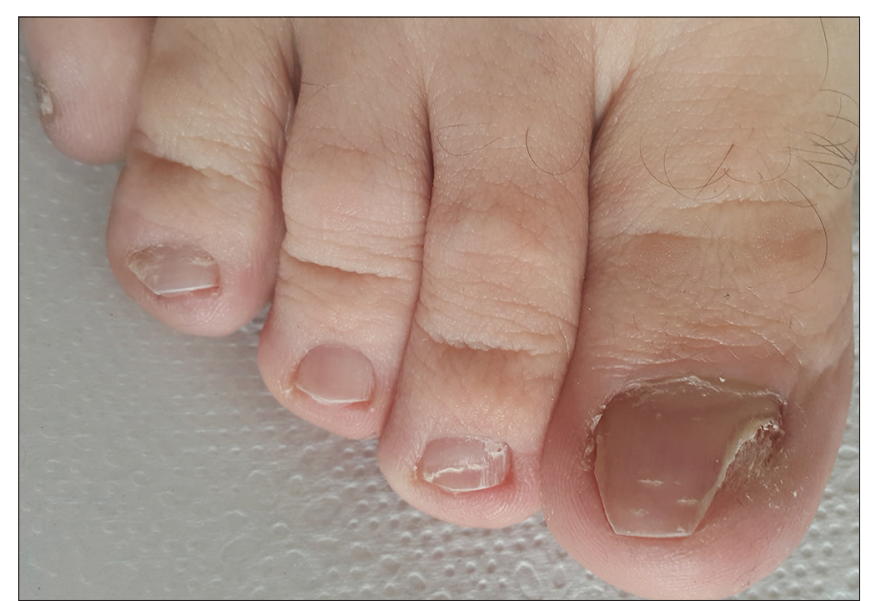

Figure 1: Subungual hyperkeratosis and onycholysis on the lateral side of the first toenail, onychoschizia of the second toenail, subungual hyperkeratosis of the fourth and fifth toenails. There is no pigmentation or discoloration of the toenail.

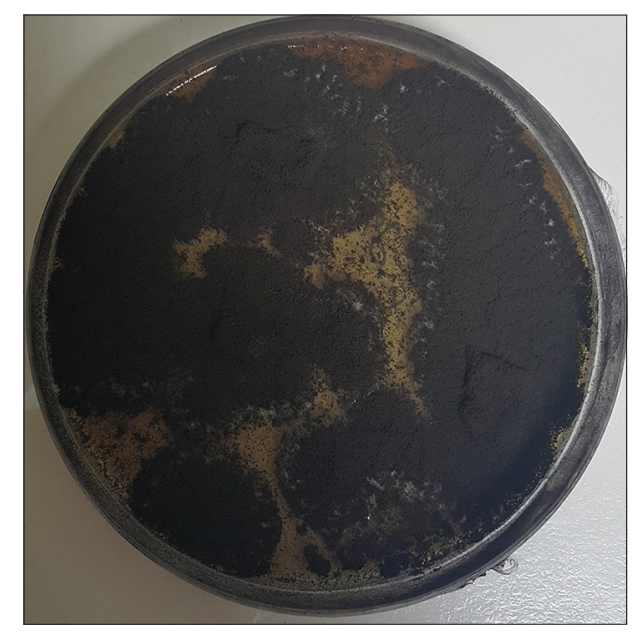

Figure 2: The mycological culture of the nail clippings of the first toenail revealed Aspergillus niger on Sabouraud's dextrose agar.

to $12 \%$ of the patients with onychomycosis $[1,2]$. Having a family member with onychomycosis,

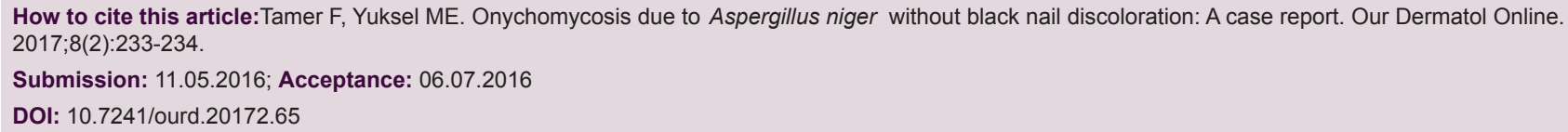


occlusive footwear, diabetes mellitus, hemodialysis, peripheral vascular disease, psoriasis, hyperhidrosis are the risk factors for onychomycosis [2]. Onychomycosis due to nondermatophytic mold is often resistant to treatment [1].

Aspergillus species have been reported as causative agents in $2.6 \%$ to $6.1 \%$ of the patients with onychomycosis [1]. In recent years, it has been suggested that Aspergillus species are identified more commonly as causative agents of onychomycosis. Nouripour-Sisakht et al. investigated 463 patients with onychomycosis. The causative agent was nondermatophyte molds in 154 (33.2\%) patients. Moreover, Aspergillus species were identified in $135(87.8 \%)$ cases of nondermatophyte molds. Aspergillus niger was detected as causative agent in only $4(2.6 \%)$ patients [3]. Wijesuriya et al. investigated 255 diabetic patients with onychomycosis. They isolated Aspergillus species in $180(71 \%)$ patients. Furthermore, Aspergillus niger was the causative agent in $76 \%$ of these patients [4].
Increased incidence of onychomycosis caused by Aspergillus niger should be kept in mind and Aspergillus niger should be considered in differential diagnosis of treatment resistant onychomycosis.

\section{REFERENCES}

1. Kim DM, Suh MK, Ha GY, Sohng SH. Fingernail onychomycosis due to Aspergillus niger. Ann Dermatol. 2012;24:459-63.

2. Moreno G, Arenas R. Other fungi causing onychomycosis. Clin Dermatol. 2010;28:160-3.

3. Nouripour-Sisakht S, Mirhendi H, Shidfar MR, Ahmadi B, Rezaei-Matehkolaei A, Geramishoar M, et al. Aspergillus species as emerging causative agents of onychomycosis. J Mycol Med. 2015;25:101-7.

4. Wijesuriya TM, Kottahachchi J, Gunasekara TD, Bulugahapitiya U, Ranasinghe KN, Neluka Fernando SS, et al. Aspergillus species: An emerging pathogen in onychomycosis among diabetics. Indian J Endocrinol Metab. 2015;19:811-6.

Copyright by Funda Tamer, et al. This is an open access article distributed under the terms of the Creative Commons Attribution License, which permits unrestricted use, distribution, and reproduction in any medium, provided the original author and source are credited.

Source of Support: Nil, Conflict of Interest: None declared. 